\title{
Sunitinib prevents cachexia and prolongs survival of mice bearing renal cancer by restraining STAT3 and MuRF-1 activation in muscle
}

\author{
Francesca Pretto ${ }^{1,4, *}$, Carmen Ghilardi ${ }^{1, *}$, Michele Moschetta ${ }^{1}$, Andrea Bassi ${ }^{2}$, \\ Alessandra Rovida ${ }^{1}$, Valentina Scarlato ${ }^{1}$, Laura Talamini ${ }^{1}$, Fabio Fiordaliso ${ }^{3}$, Cinzia \\ Bisighini $^{3}$, Giovanna Damia ${ }^{1}$, Maria Rosa Bani ${ }^{1}$, Rosanna Piccirillo ${ }^{1}$ and Raffaella \\ Giavazzi ${ }^{1}$ \\ ${ }^{1}$ Department of Oncology, IRCCS-Istituto di Ricerche Farmacologiche "Mario Negri", 20156 Milan, Italy \\ 2 Department of Phisics, Politecnico di Milano, 20133 Milan, Italy \\ 3 Department of Cardiovascular Research, IRCCS-Istituto di Ricerche Farmacologiche "Mario Negri", 20156 Milan, Italy \\ ${ }^{4}$ Present address: Philochem AG, 8112 Otelfingen, Switzerland \\ * These authors contributed equally to this work \\ Correspondence to: Raffaella Giavazzi, email: raffaella.giavazzi@marionegri.it \\ Rosanna Piccirillo, email: rosanna.piccirillo@marionegri.it
}

Keywords: renal carcinoma, cancer cachexia, muscle wasting, sunitinib, STAT3, xenograft model

Received: October 15, $2014 \quad$ Accepted: November 14, $2014 \quad$ Published: November 15, 2014

This is an open-access article distributed under the terms of the Creative Commons Attribution License, which permits unrestricted use, distribution, and reproduction in any medium, provided the original author and source are credited.

\section{ABSTRACT}

Tyrosine kinase inhibitors, affecting angiogenesis, have shown therapeutic efficacy in renal cell carcinoma (RCC). The increased overall survival is not fully explained by their anti-tumor activity, since these drugs frequently induce disease stabilization rather than regression. RCC patients frequently develop cachectic syndrome. We used the RXF393 human renal carcinoma xenograft that recapitulates the characteristics of the disease, including the growth in the mouse kidney (orthotopic implantation), and the induction of cachexia with subsequent premature death. Sunitinib prevents body weight loss and muscle wasting and significantly improves the survival of RXF393-bearing nude mice. The anti-cachectic effect was not associated to direct anti-tumor activity of the drug. Most relevant is the ability of sunitinib to reverse the cachectic phenotype and rescue the animals from the loss of fat tissue. Body weight loss is prevented also in mice bearing the C26 colon carcinoma, classically reported to induce cachexia in immunocompetent mice. Among the mechanisms, we herein show that sunitinib is able to restrain the overactivation of STAT3 and MuRF-1 pathways, involved in enhanced muscle protein catabolism during cancer cachexia.

We suggest that off-target effects of angiogenesis inhibitors targeting STAT3 are worth considering as a therapeutic option for patients who develop cachexia, independently of their anti-tumor activity.

\section{INTRODUCTION}

Cachexia is a dramatic wasting syndrome associated with several chronic diseases, including cancer, and primarily involves loss of muscle mass [1]. Cancer cachexia affects up to $80 \%$ of cancer patients and causes reduced physical function, low tolerance to anti-cancer therapy and shorter survival [1, 2]. Muscle wasting results from excess of protein catabolism over synthesis and during cancer it is triggered by increased levels of proinflammatory cytokines [3, 4]. Given the complexity of this syndrome, progress in the treatment of cancer cachexia has been slow. Cancer cachexia is an important unmet medical need for which multimodal management is 
normally aimed at the best supportive care $[5,6]$.

Patients afflicted by renal cell carcinoma (RCC) frequently develop cachexia [7]. This syndrome is believed to be caused by the secretion of cytokines or hormones from the tumor or the immune system [8]. Tumor-specific growth factors, like vascular endothelial growth factor (VEGF) and platelet-derived growth factor (PDGF), are also highly secreted by RCC [8]. In the past five years, the introduction of targeted anti-angiogenic therapy has dramatically enlarged the number of therapeutic options for the treatment of RCC and significantly improved the prospects for patients [9].

Sunitinib is a multi-targeted receptor tyrosine kinase inhibitor (TKI) that mainly targets VEGF and PDGF receptors and it was the first oral TKI to gain regulatory approval in this setting [10]. Sunitinib is the reference standard of care, recommended in international guidelines for the first-line treatment of favorable- or intermediaterisk RCC [11]. The range of agents available for the treatment of RCC has expanded substantially, including sorafenib, temsirolimus, everolimus, bevacizumab in combination with interferon- $\alpha$ and, more recently, pazopanib and axitinib [12]. However, while increasing overall survival, these targeted drugs frequently induce only disease stabilization. Since survival benefits in RCC patients do not always correlate with tumor response, other unknown mechanisms may account for the clinical benefits.

In the present study, we show that mice bearing RCC-derived RXF393 develop cachexia (i.e. body weight loss), which is prevented and/or reversed upon treatment with sunitinib, resulting in increased survival. Both muscle and fat tissues undergo wasting in RXF393-bearing mice and sunitinib is able to prevent the loss of both. This unprecedented therapeutic effect is not associated with its anti-tumor activity and involves prevention of STAT3 activation and MuRF-1 over-expression in muscles. As a consequence, STAT3 inhibitors should be worth evaluating as a therapeutic option for cancer patients who develop cachexia.

\section{RESULTS}

\section{Sunitinib prevents RXF393-induced cachexia}

The human renal carcinoma RXF393 transplanted in nude mice forms a rapidly growing tumor causing progressive body weight loss (BWL), typical of the cachectic syndrome. As shown in Figure 1A, all RXF393bearing mice rapidly lost weight (a hallmark of cachexia) around 15 days after subcutaneous tumor transplantation, when tumor weight (TW) was about $700 \mathrm{mg}$, and died shortly after. A significant inverse correlation was observed between tumor and body weight $(\mathrm{R}=-0.941, \mathrm{P}<0.001)$.
When tumor-bearing mice were treated with sunitinib, the inverse correlation between tumor and body weight was lost, but it was rapidly restored when treatment was interrupted (Fig.1B). Sunitinib treatment (started at about $100 \mathrm{mg}$ of TW) had a moderate, but significant anti-tumor effect (T/C 30\%) (Fig.1C, left). All the RXF393-bearing mice treated with vehicle developed cachexia (BWL > $20 \%$ ) and ought to be killed within 23 days after tumor transplantation (Fig.1C, middle), whereas the overall survival (time of sacrifice) of sunitinib-treated mice was significantly higher (MST 51 days; ILS 250\%) (Fig.1C, right) and, surprisingly, the tumor reached the maximal ethically accepted weight $(<2000 \mathrm{mg})$ without causing cachexia. At autopsy, subcutaneous tumors harvested from sunitinib-treated mice showed a pale phenotype, compared to the highly vascularized appearance of the vehicle-treated tumors, consistent with the anti-vascular activity of sunitinib (Fig.1C, inserts). Immunohistological analysis confirmed the lower vessel density in sunitinibtreated tumors (data not shown).

To exclude that the anti-cachectic effects of sunitinib depends on its effect on tumor growth, we compared the therapeutic active dose of sunitinib $(40 \mathrm{mg} / \mathrm{kg})$ to suboptimal doses (20 and $10 \mathrm{mg} / \mathrm{kg}$ ) in RXF393-bearing mice. As shown in Fig.1D, left, $40 \mathrm{mg} / \mathrm{kg}$ inhibited tumor growth, while doses of $20 \mathrm{mg} / \mathrm{kg}$ or $10 \mathrm{mg} / \mathrm{kg}$ did not exert anti-tumor effects. However, a dose-response effect on BWL was observed: $10 \mathrm{mg} / \mathrm{kg}$ delayed BWL by four days and $20 \mathrm{mg} / \mathrm{kg}$ was able to preserve body mass (Fig.1D, right). These results suggest that the effect on cachexia by sunitinib involves mechanisms aside from inhibition of angiogenesis and tumor growth.

To confirm the anti-cachectic effects of sunitinib in a more relevant setting, RXF393 was transplanted orthotopically in the kidney of nude mice (Fig.2). Tumorbearing mice started losing body weight in three weeks (Fig.2A), and were to be sacrificed (MST 30 days, Fig.2B) when their tumorgrafts reached about $600 \mathrm{mg}$ (Fig.2C), because of clear signs of discomfort (i.e. kyphosis, immobility, tremor; $20 \%$ of BWL). In contrast, sunitinibtreated mice lived significantly longer (MST 51 days; ILS $70 \%$, Fig. $2 \mathrm{~B}$ ) and no sign of BWL was observed when mice were sacrificed with a tumor mass of approx. $>1 \mathrm{~g}$, (Fig.2A and C). At autopsy, tumors growing in the kidney of sunitinib-treated mice had a pale phenotype, consistent with the drug's activity (Fig.2C).

\section{Sunitinib prevents RXF393-induced muscle wasting}

One of the key features of cancer-induced wasting syndrome is the loss of skeletal muscle mass [13]. To test whether cachexia was accompanied by skeletal muscle atrophy, and whether sunitinib prevented this, mice bearing subcutaneous RXF393 were treated with sunitinib or vehicle and muscle wasting was analyzed at autopsy. 
To exclude differences due to the tumor burden, RXF393bearing mice from vehicle- and sunitinib-treated groups were sacrificed when tumor reached comparable sizes.

Comparable tumor weights (about 400mg) were associated to significant BWL in vehicle-treated mice, whereas the body weight of sunitinib-treated ones were undistinguishable from age- and sex-matched healthy mice (tumor-free) (Fig.3A). After death, heart, tibialis anterior (TA) and gastrocnemius muscles were collected and weighed. In RXF393-bearing mice, no loss of heart weight was observed both in vehicle- and sunitinibtreated mice (Fig.3B) compared to healthy mice, while the weight of TA and gastrocnemius muscles from vehicletreated ones decreased by about 25\% (Fig.3C). Strikingly, the mean weight of both muscles from sunitinib-treated mice did not significantly differ from that of healthy mice, indicating that sunitinib completely protects from RXF393-induced muscle wasting (Fig.3C). Moreover, the cross-sectional area (CSA) of the TA myofibers showed a significant reduction between vehicle-treated and healthy animals (1122 $\mu \mathrm{m}^{2}$ and $1789 \mu \mathrm{m}^{2}$, respectively), which was partially but significantly restored upon sunitinib treatment (1432 $\mu^{2}$ ) (Fig.3D, left). Accordingly, electron microscopy ultra-structural analysis of the TA muscles of healthy mice showed the expected crosssectional organization of thin and thick filaments in myofibrils, surrounded by mitochondria and cisternae of the sarcoplasmic reticulum. Conversely, cachectic muscles from tumor-bearing mice showed derangement and degeneration of both types of filaments, while
A

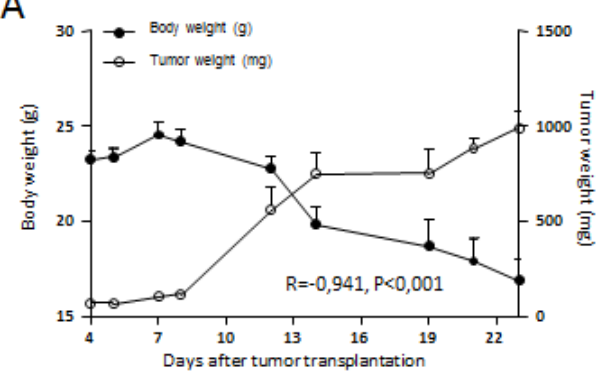

C
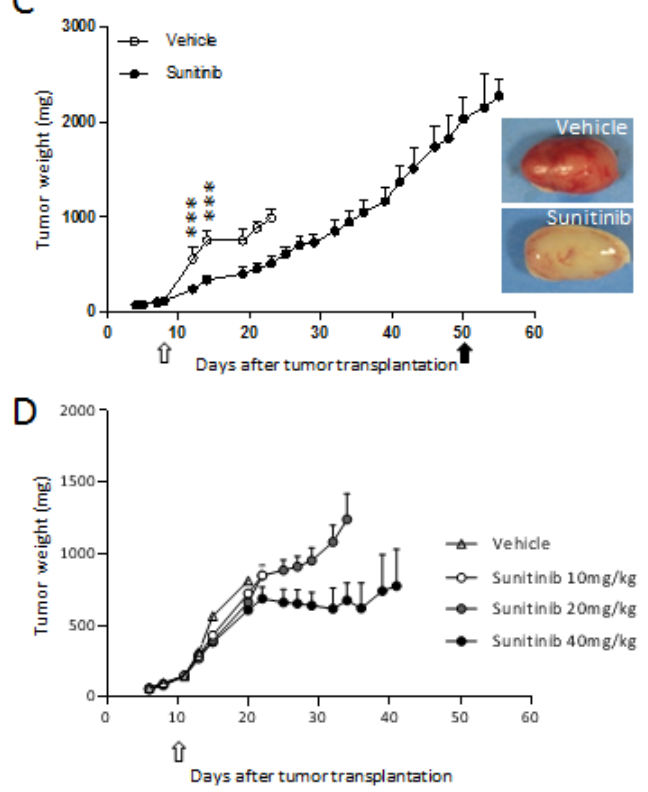
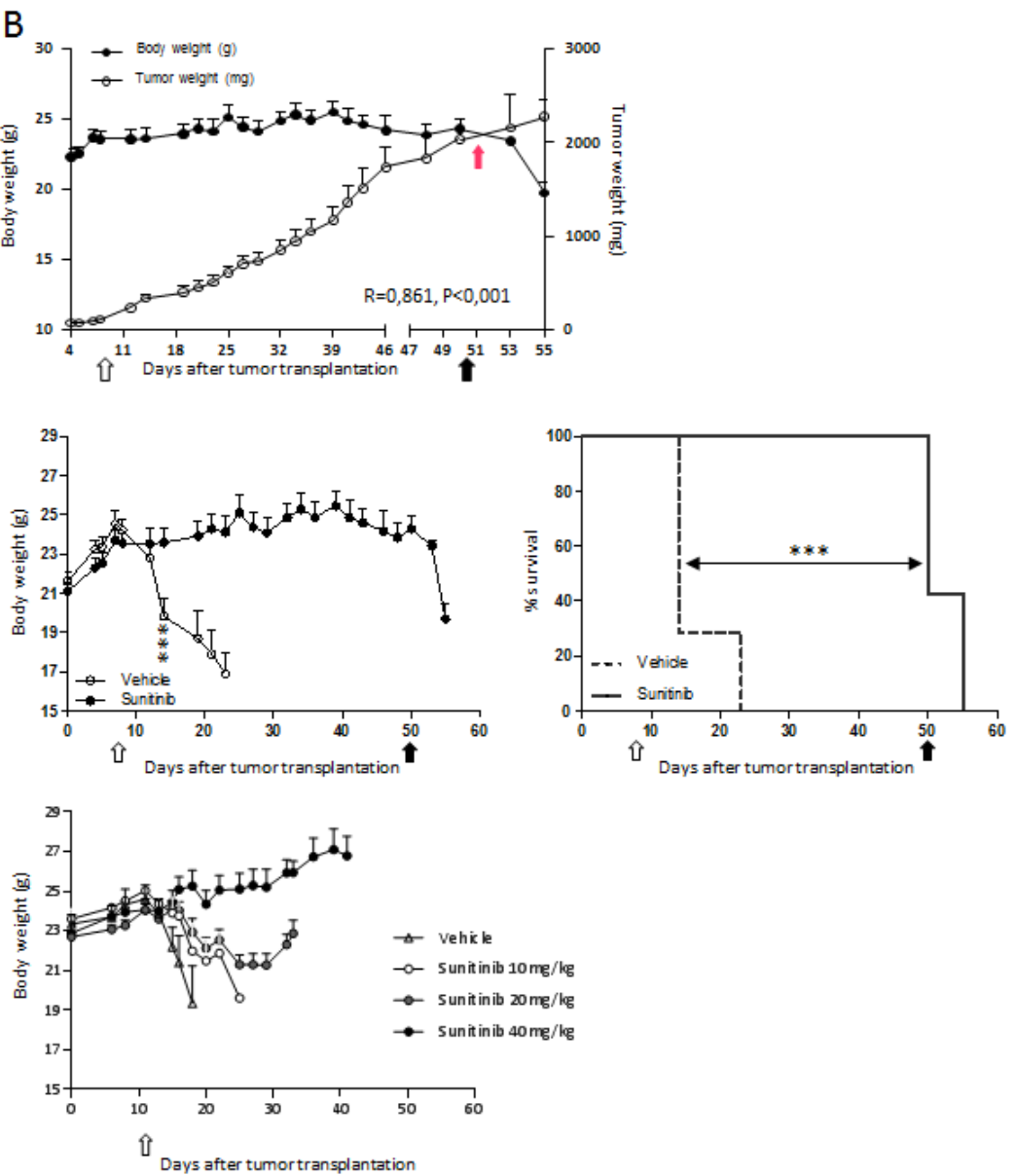

Figure 1: RXF393-induced cachexia is prevented by sunitinib treatment independently from tumor inhibition. A, RXF393 cells were injected subcutaneously into the flank of nude mice: tumor and body weight are plotted over time and their correlation is shown. B, RXF393-bearing mice were randomized (TW $120 \mathrm{mg}, \mathrm{n}=7 /$ group) to receive sunitinib (40 mg/kg, p.o.) daily for the indicated time (arrows): tumor and body weights are reported and their correlation is shown. C, RXF393-bearing mice were randomized (TW 120 $\mathrm{mg}, \mathrm{n}=7$ /group) to receive sunitinib or vehicle daily for the indicated time (arrows). Tumor weights (left panel) and representative images (inserts) of the gross appearance of treated and untreated tumors, excised when mice were euthanized, are shown. Body weights are plotted over time (middle panel). Mice treated with sunitinib or not were sacrificed when they lost $20 \%$ of body weight and/or showed signs of distress (loss of mobility, kyphosis, tremor). Survival curves are depicted (right panel). D, RXF393-bearing mice were randomized (TW $150 \mathrm{mg}, \mathrm{n}=9 /$ group) to receive vehicle or sunitinib at the doses of $10,20 \mathrm{or} 40 \mathrm{mg} / \mathrm{kg}$ daily p.o. The effects of sunitinib on tumor growth (left panel) and BW (right panel) are reported. Open arrow=treatment starts, full arrow=treatment ends, red arrow $=$ day of inversion. 
mitochondria and sarcoplasmic reticulum retained their normal morphology. Interestingly, treatment of cachectic mice with sunitinib reversed the abnormal morphology of TA, restoring the normal architecture of sarcomeres (Fig.3D, right).

\section{Sunitinib prevents C26-induced cachexia}

The beneficial effects of sunitinib were further confirmed in another experimental model fully characterized for cancer cachexia and previously used to study anti-cachexia interventions: the murine C26 colon carcinoma [14].

As expected, the C26 tumor induced about 5\% BWL already 11 days after transplantation and, when BWL reached $20 \%$, it led to death $90 \%$ of the vehicle-treated mice within 15 days (Fig.4A). Likewise RXF393 model, there was a significant inverse correlation between tumor and body weight $(\mathrm{R}=-0.9, \mathrm{P}<0.05)$ (Fig.4A).

Sunitinib only minimally inhibited the tumor growth (T/C 69\%) (Fig.4B), but it significantly delayed the BWL compared to vehicle-treated mice (Fig.4C, left). However, when the treatment was interrupted at day 20, all the mice previously treated with sunitinib lost weight and were terminally euthanized within one week (Fig.4C).

To exclude that the lower BWL in sunitinib-treated mice was caused by smaller tumors due to treatment, we compared mice bearing tumors of similar size (about $280 \mathrm{mg}$ ), irrespectively of the time after transplantation and found that the body weight of sunitinib-treated was significantly higher than that of vehicle-treated mice (Fig.4D).

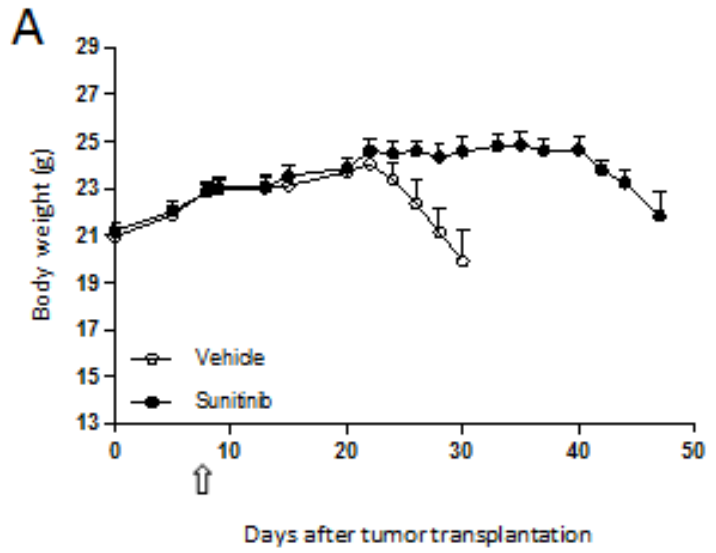

B
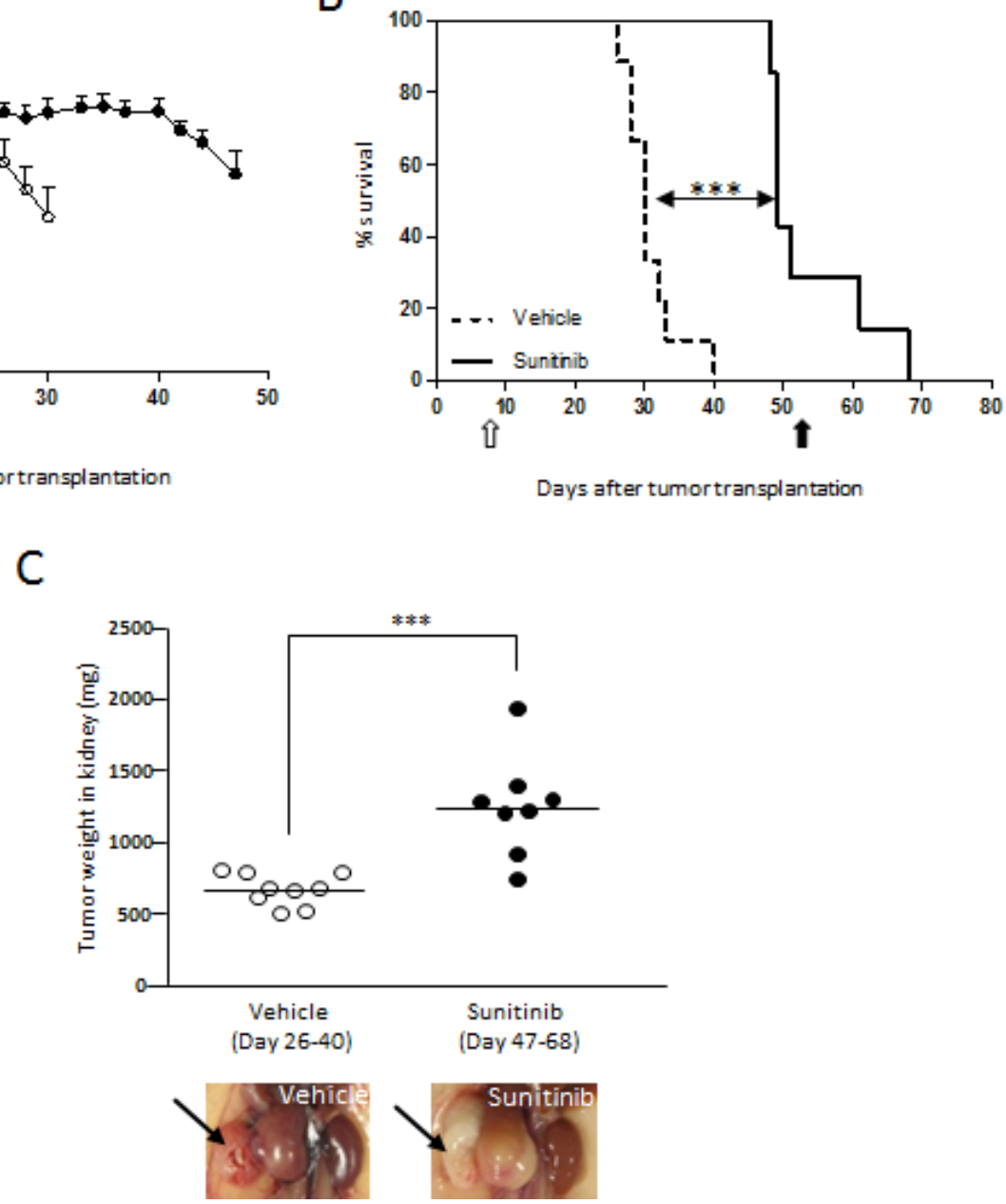

Figure 2: Cachexia is prevented by sunitinib treatment in the RXF393 orthotopic model. RXF393 cells were injected orthotopically into the right kidney of nude mice and growing tumors were treated with sunitinib (40 $\mathrm{mg} / \mathrm{kg}$, p.o.) or vehicle daily for the indicated time ( $\mathrm{n}=9$ /group). A, Body weights are plotted over time. B, Mice treated with sunitinib or not were sacrificed when they lost $20 \%$ of body weight and/or showed signs of distress (loss of mobility, kyphosis, tremor) and survival curves are shown. C, Tumor weights were determined at sacrifice and median is reported. Range day of sacrifice is reported in brackets. Representative images of the gross appearance of treated and untreated tumors growing in the kidney are shown in the inserts. Open arrow=treatment starts, full arrow=treatment ends. 


\section{Sunitinib reverses RXF393-induced cachexia}

To test the ability of sunitinib to reverse the wasting syndrome once established, mice bearing RXF393 and developing cachexia were randomized to receive sunitinib or vehicle, when BWL was around $10 \%$ (14 days after tumor implantation). As shown in Fig.5A, sunitinib completely and rapidly reversed BWL even when treatment started with larger tumors (about 600-700 mg, Fig.5B). The recovery in body weight was accompanied by a gain in fat tissue, as shown by micro-CT (computed tomography) scans of the entire abdominal region of tumor-bearing mice treated with sunitinib (Fig.5C and D, day 24). Vehicle-treated mice lost fat tissue concomitantly to BWL (day 16). As expected, soon after suspension of sunitinib treatment, mice started to lose weight (Fig.5A) and fat tissue (Fig.5C, day 33) at a level comparable to untreated mice. Of note, body mass was not significantly affected in tumor-free mice treated or not with sunitinib (data not shown). Altogether, these data show that sunitinib treatment exerts its effects, such as preservation of body mass and fat tissue, as long as its administration is maintained, and even if cachexia has already advanced.
A

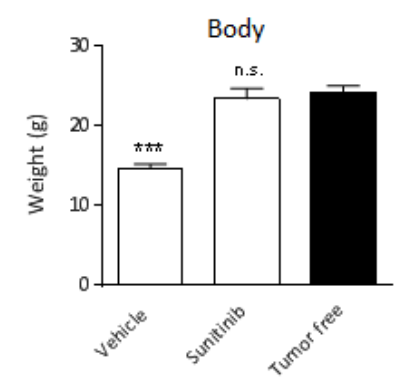

C

Tibialis anterior

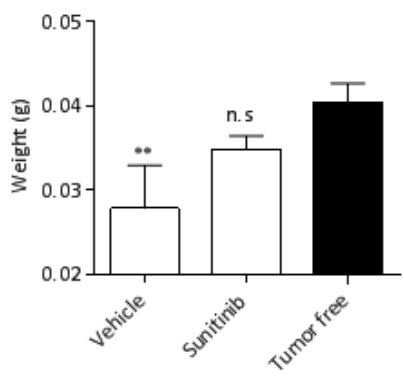

B

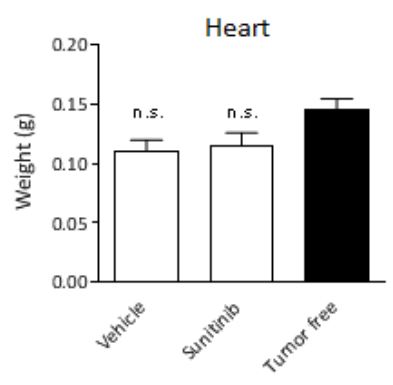

Gastrocnemius

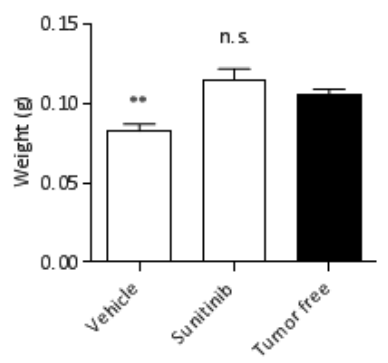

D

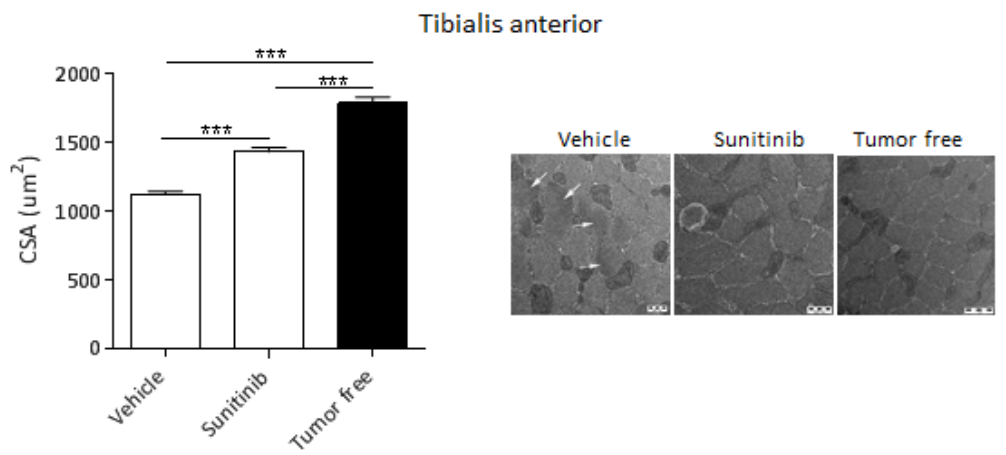

Figure 3: Sunitinib prevents RXF393-induced skeletal muscle wasting. RXF393-bearing mice were randomized (TW 120 $\mathrm{mg}, \mathrm{n}=7 /$ group) to receive sunitinib ( $40 \mathrm{mg} / \mathrm{kg}$ p.o.) or vehicle daily. Animals were sacrificed at tumor sizes of about $400 \mathrm{mg}$, and bodies (A), hearts (B), TA and gastrocnemius muscles (C) were weighed. D, The effects of treatment on the TA muscles were also determined by quantitation of the CSA. Representative ultrastructural images by electron microscopy, showing the morphology of the TA in the various conditions are provided. Results are plotted as mean \pm SD. n.s. $=$ not significant. 

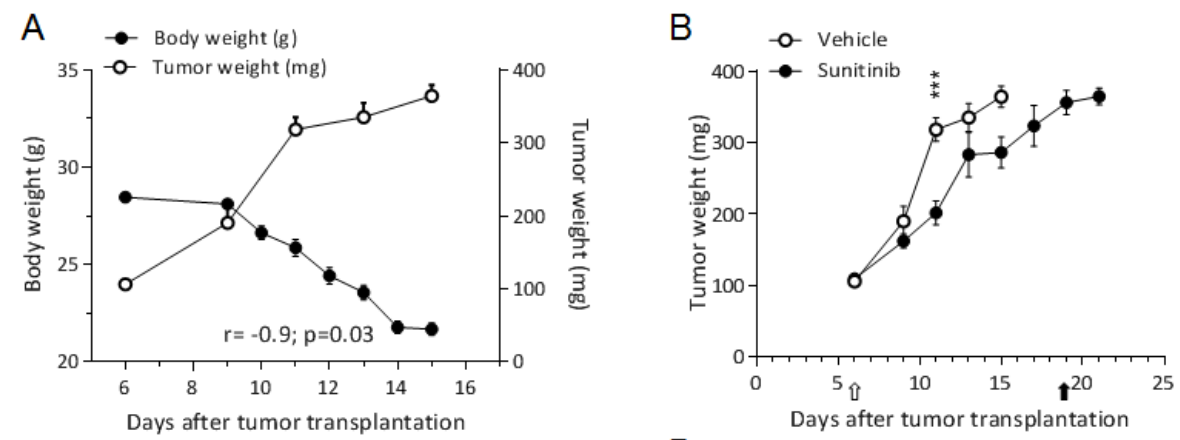

C

D
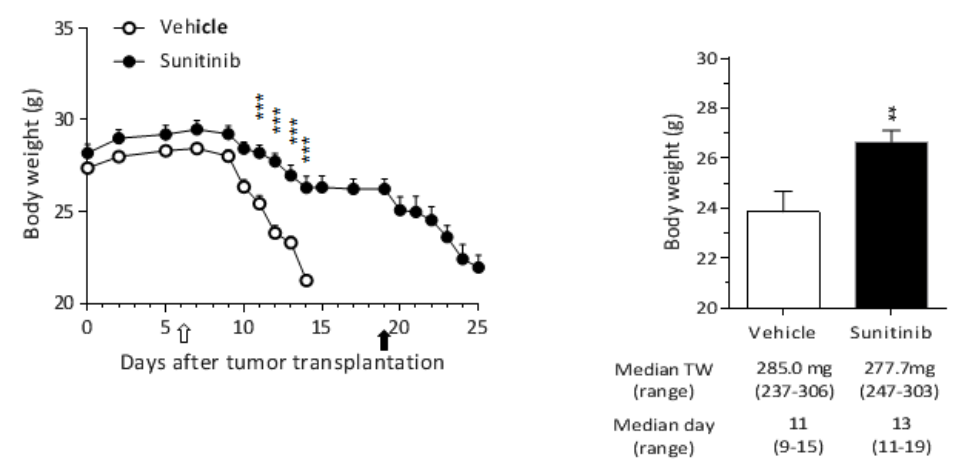

Figure 4: Sunitinib prevents C26-induced cachexia. A, C26 cells $\left(1 \times 10^{6}\right)$ were inoculated subcutaneously in the upper right flank of BALB/c mice. Tumor and body weight are plotted over time and their correlation is shown. B-C, C26-bearing mice were randomized (TW $100 \mathrm{mg}$ ) to receive sunitinib (40 mg/kg, p.o.) or vehicle ( $\mathrm{n}=12$ /group) daily for the indicated time; tumor growth (B) and body weight (C) are reported. Open arrow $=$ treatment starts, full arrow $=$ treatment ends. D, Body weight of animals with tumors at the same size (approx. $280 \mathrm{mg}$, irrespectively of the time after tumor transplantation) ( $\mathrm{n}=7 /$ group) is reported. Median tumor weight and day with range are reported.
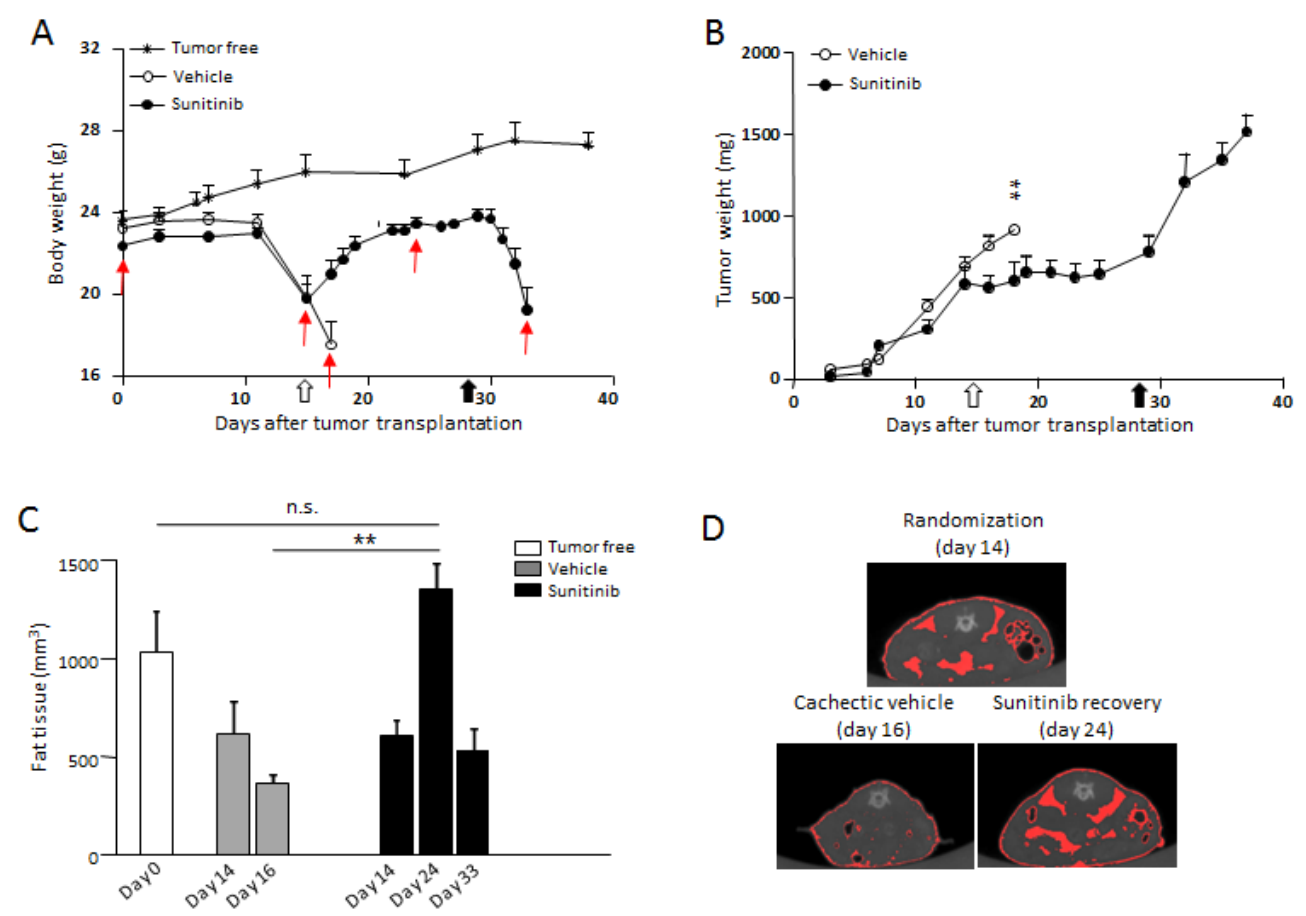

Figure 5: Sunitinib reverses RXF393-induced cachexia. Mice bearing subcutaneously RXF393 were randomized with a cachectic phenotype, (10\% BWL and initial signs of discomfort) and treated with sunitinib (40mg/kg p.o.) or vehicle daily for two weeks ( $\mathrm{n}=7-9$ / group). A-B, The effects of sunitinib on body and tumor weights are shown in A and B, respectively. C-D, The effect of sunitinib on fat content was determined by micro-CT scan. A quantitation of abdominal fat at the indicated times is reported (C). Data are plotted as mean $\pm \mathrm{SD}(\mathrm{n}=5)$. Representative images from mice at randomization or from vehicle- or sunitinib-treated mice are shown and the fat tissue is highlighted in red (D). Open arrow=treatment starts, full arrow=treatment ends, red arrows=day of CT analysis. 


\section{Sunitinib prevents MuRF-1 and STAT3 activation in muscle of RXF393-bearing mice}

Loss of skeletal muscle mass is generally due to reduced protein synthesis, increased degradation or a relative imbalance of the two [3,4]. The molecular pathways implicated in these mechanisms were investigated in TA muscles of vehicle- and sunitinibtreated mice. The Muscle RING Finger 1 protein (MuRF1), a muscle-restricted ubiquitin ligase involved in the accelerated protein degradation during various kinds of muscle atrophy [15], was found highly up-regulated in muscles from cachectic RXF393-bearing mice, while sunitinib treatment was able to prevent MuRF-1 upregulation (Fig.6A). More prolonged sunitinib treatment led to down-regulation of atrogin-1, another musclespecific ubiquitin ligase triggering muscle atrophy [15, 16], although the effect was less evident. Levels of
p-AKT over total AKT, usually considered as index of protein synthesis, were also evaluated, but not significant difference was detected (data not shown).

An important mediator implicated in the development of cancer cachexia is the transcription factor STAT3 that previous work has shown activated by circulating pro-inflammatory cytokines (i.e. IL-6) [17]. Muscles from cachectic RXF393-bearing mice exhibited over-activation of STAT3, as shown by higher levels of phosphorylated STAT3 (p-STAT3) compared to noncachectic ones (analyzed at randomization) (Fig.6B). Sunitinib inhibited p-STAT3 of muscles from RXF393bearing mice at levels comparable to non-cachectic mice.

Similar results were obtained with sorafenib, another multitargeted TKI, also used for the treatment of renal cancer [18]. Administration of sorafenib prevented the body and muscle weight loss in RXF393-bearing mice and this was associated with the inhibition of STAT3 and
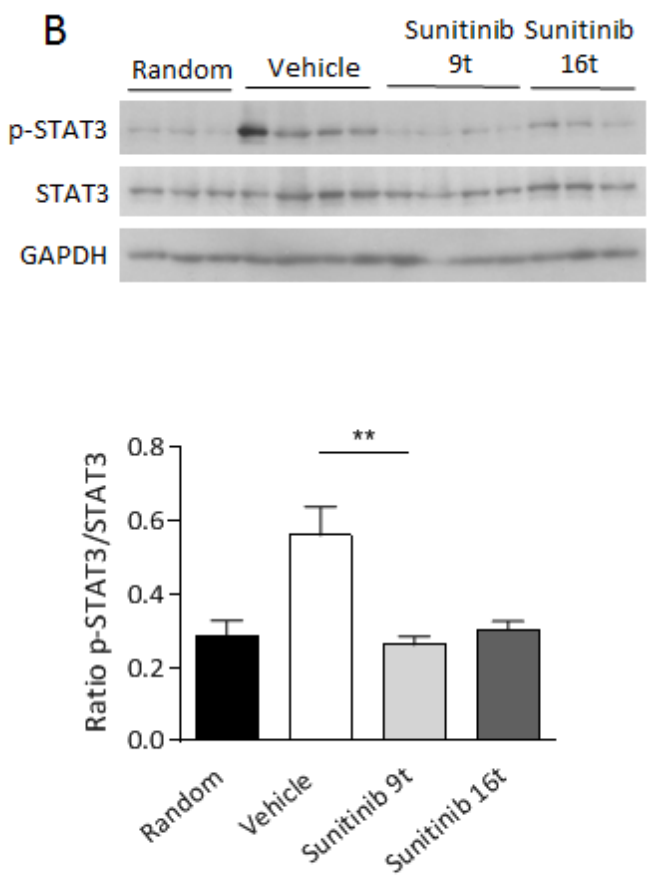

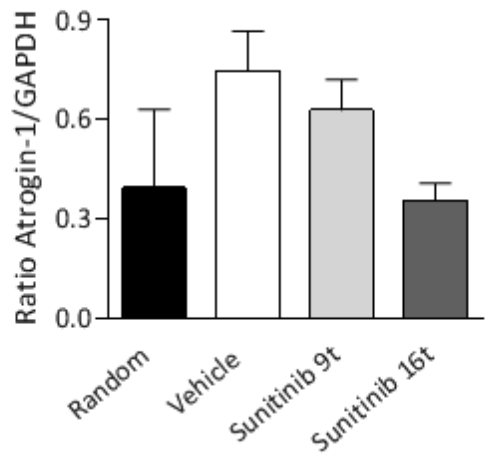

Figure 6: Skeletal muscle wasting is prevented by sunitinib through inhibition of MuRF-1 and p-STAT3. A-B, Tibialis anterior muscles of untreated or sunitinib-treated mice (after 9 (9t) or 16 (16t) days of treatments) were analyzed by Western Blot for MuRF-1, atrogin-1 (A), p-STAT3 and STAT3 (B) protein levels. Tibialis anterior of non-cachectic mice, sacrificed at randomization, were chosen as reference (indicated as Random). GAPDH was used as internal loading control. Densitometric analysis was performed and quantitation of protein levels is provided. Results are plotted as mean $\pm \mathrm{SD}$. 
MuRF-1 pathways in TA muscles (Supplementary Fig.1).

IL-6 was elevated in plasma of mice bearing RXF393 or C26, but sunitinib did not change its levels in neither model (Supplementary Fig.2), suggesting that the effect of sunitinib is not mediated by the direct attenuation of this circulating cytokine.

Overall, these data indicate that RXF393-bearing mice offer a unique model to test drugs against cancer cachexia and that sunitinib, as well as sorafenib, can prevent cachexia in vivo by lowering protein catabolism through inhibition of STAT3/MuRF-1 activation at least in muscles.

\section{DISCUSSION}

Renal cell carcinoma is one of the malignancies that mostly causes a cancer-associated systemic syndrome (i.e. cachexia), mainly consisting in progressive loss of the body energy stores and likely reflecting the high production of cytokines and growth factors [7, 8]. Herein, we report that treatment with sunitinib (and sorafenib, Supplementary data) prolonged the survival of mice bearing the human kidney carcinoma RXF393, transplanted either ectopically in the subcutis or orthotopically in the kidney (i.e. mimicking the site of tumor origin), by blocking the BWL (i.e. muscle and fat wasting) caused by cancer growth.

Unexpected was the ability of sunitinib to reverse the cachectic phenotype once established. In fact, sunitinib reversed BWL and rescued the animal from the loss of abdominal fat tissue. The anti-cachectic effects of sunitinib are not associated to reduced tumor growth, as revealed by the prevention of cachexia also at sub-optimal doses of sunitinib that did not inhibit tumor growth. Importantly, BWL was prevented also in a syngeneic tumor model, the C26 colon cancer, classically reported to induce cachexia in immunocompetent mice [19]. This shows that such anti-cachectic effect also occurs with an intact immune system and, furthermore, implies that it is not restricted to a single tumor model. These results might explain the clinical evidence that often targeted drugs, including sunitinib, increase overall survival of RCC patients, without inducing tumor shrinkage, but rather causing disease stabilization $[18,20]$.

Of note, during human RCC both cancer and subsequent kidney dysfunction may account for the cachectic appearance, further accelerating muscle loss [21]. Our data, showing similar rates of BWL in mice bearing a tumor subcutaneously or orthotopically in the kidney, possibly exclude that kidney failure may worsen the RXF393-related cachexia.

We have investigated the anti-cachectic effect exerted by sunitinib not only at the macroscopic level of multiple tissues (fat, heart and skeletal muscles) but also at the ultrastructural level in skeletal muscle (i.e. the most affected tissue in cancer cachexia). Surprisingly, we observed that sunitinib not only was able to spare the muscle mass but even the misalignment of myofibers induced by cancer progression. As reported by Aulino and coworkers [19] in C26-bearing mice, we report that cachectic muscles from RXF393 mice display an aberrant distribution of thin and thick filaments that may be due to selective degradation of muscle proteins [22]; these aberrations can be prevented by sunitinib, allowing preservation of muscle mass and probably of its function.

Decreased protein synthesis and increased proteolysis are among the mechanisms leading to muscle loss [3]. During atrophy, MuRF-1 and atrogin-1 are the crucial muscle-specific ubiquitin ligases that direct the polyubiquitination of proteins to target them for proteolysis by the $26 \mathrm{~S}$ proteasome, mediating sarcomeric breakdown (MuRF-1, which degrades myosins) [23] or shifting gene expression towards a less myogenic phenotype (atrogin-1, which degrades MyoD) [24]. Our results indicate that cachexia prevention by sunitinib is mainly determined by the reduction of proteolysis, rather than increased synthesis, as shown by suppression of MuRF-1 and, though less evident, atrogin-1 induction and unchanged levels of p-AKT/AKT ratio. Since FoxO3 is a master transcription factor driving muscle wasting by upregulating both MuRF-1 and atrogin-1 [25, 26], we have also measured the levels of p-FoxO3 over total FoxO3 in the vehicle and sunitinib-treated muscles, but no difference was detected. Instead, our data suggest that improvement of cachexia is associated to STAT3 inhibition in muscles by sunitinib.

Cancer cachexia is triggered by increased systemic inflammation, where pro-inflammatory cytokines (e.g. IL6) play a major role [27]. Previous reports demonstrated that STAT3 activation induced by IL-6 is per se sufficient to induce muscle fiber wasting in vitro as well as in vivo and that STAT3 inhibition would abolish skeletal muscle wasting downstream of IL-6 in cancer cachexia models $[17,28]$. Interestingly, STAT3 has been found activated also in wasting muscles from patients suffering from chronic kidney disease [29] and very recently involved in the control of satellite cell expansion and muscle repair [30]. Moreover, the IL-6/STAT3 pathway has been also implied in increased lipolysis of C26-bearing mice [31], thus suggesting that in our experimental models sunitinib may spare fat and muscle tissues through similar targets. We found that circulating IL-6 was elevated in both nude mice bearing RXF393 and in immunocompetent mice bearing $\mathrm{C} 26$, (as described by others $[32,33]$ ), but was not reduced by sunitinib (Supplementary Fig.2). This indicates that the anti-cachectic effect of sunitinib is not due to reduced circulating IL-6 or that the inhibitory target of sunitinib is downstream of the IL-6 cascade (i.e. JAK) [17]. Similar results were found upon treatment of cachectic rodents with anti-myostatin inhibitors, where signs of cachexia were reversed without affecting IL-6 or TNF- $\alpha$ plasma levels [32]. 
The RXF393 xenograft model secretes high levels of VEGF, likely enough to cause a cachectic syndrome [34, 35]. Indeed, Cao et coworkers have shown that systemic treatment with anti-VEGF agents reversed VEGF-induced cancer associated systemic syndrome and prolonged mouse survival [34]. Surprisingly, antibodies anti-VEGF (bevacizumab) or anti-VEGF receptor 2 (DC101) did not protect RXF393-carrying mice from cachexia to the same extent as sunitinib (data not shown). Presumably, other cytokines or growth factors are responsible for cachexia in our tumor model.

Although our understanding of cancer cachexia has improved dramatically in the past few years, guidelines for the prevention and treatment of cancer-related cachexia are lacking. New treatments with myostatin inhibitors, thalidomide, selective COX-2 inhibitors, ghrelin mimetics and selective androgen receptor modulators have shown promising results, but their efficacy need to be confirmed in clinical trials that are at present testing multimodal interventions against cancer cachexia $[5,6]$.

Overall, we show that TKIs, such as sunitinib, prevent BWL and preserve muscle and fat tissue, by inhibiting STAT3 phosphorylation. STAT3 has been identified as a novel tumor target and several inhibitors are under development [36]. Only very recently, its pharmacological inhibition has been shown to be beneficial against cancer cachexia in experimental models [37]. Our results further encourage to regard STAT3 inhibitors as therapeutic option for cancer patients who develop cachexia, independently of their direct anti-tumor activity.

\section{MATERIAL AND METHODS}

\section{Cell lines}

RXF393 (VHL-WT) kidney carcinoma cell line [33], was obtained from the NCI Tumor Repository and cultured in RPMI1640 (Gibco) with 10\% FBS (Sigma Aldrich). Authentication of the cell line was done using the AmpFlSTR ${ }^{\circledR}$ Identifiler ${ }^{\circledR}$ PCR Amplification Kit (Applied Biosystems). Murine C26 colon cancer cells [19] were a kind gift of Mario Paolo Colombo (IRCCSIstituto Nazionale dei Tumori, Milan) and cultured in DMEM high glucose (Gibco) with 10\% FBS and 1mM L-glutamine (Gibco). Stocks of the cell lines were stored frozen in liquid nitrogen, and kept in culture for no more than six passages before injection in mice.

\section{Mice and tumor models}

RXF393 cells were injected subcutaneously (s.c., $1.5 \times 10^{6}$ cells $)$ into the flank or orthotopically ( $1 \times 10^{5}$ cells $)$ into the right kidney of six- to eight-week-old female
NCr-nu/nu mice (Harlan), as previously described [38]. Nude mice were maintained under specific pathogen-free conditions and handled using aseptic procedures. C26 cells $\left(1 \times 10^{6}\right)$ were inoculated s.c. in the upper right flank of male BALB/c mice (Harlan).

Tumor growth s.c. was measured twice a week with Vernier Caliper and plotted as mean tumor weight $( \pm \mathrm{SE})$ against days after tumor transplantation. The efficacy of treatment was expressed as best tumor growth inhibition $[\% \mathrm{~T} / \mathrm{C}=$ (median weight of treated tumors $/$ median weight of control tumors $) \times 100$ ]. In the orthotopic model, tumor appearance was checked twice a week by palpation. Cachexia was followed by recording body weight that was plotted as the mean $( \pm \mathrm{SE})$ against days after tumor transplantation. Mice were sacrificed when BWL reached $>20 \%$ and/or the animals showed clear signs of distress (ruffled fur for BALB/c mice, tremor, loss of mobility, kyphosis), however with a tumor burden $<2$ g. Survival day was the time at which mice were euthanized. The increment of life span (ILS) was calculated as 100x[(median survival day of treated mice - median survival day of vehicle treated mice)/ median survival day of vehicle treated]. Animal study management and data collection were done with the Study Director 1.8 software (Studylog System, Inc., San Francisco, USA) connected to a digital caliper and an electronic balance.

Procedures involving animals and their care were conducted in conformity with institutional guidelines in compliance with national (Legislative Decree $\mathrm{n}$. 26, March 4, 2014; Authorization n.19/2008-A issued March 6, 2008, by the Italian Ministry of Health) and international laws and policies (EEC Council Directive 2010/63, August 6, 2013; Standards for the Care and Use of Laboratory Animals, U.S. National Research Council, Statement of Compliance A5023-01, October 28, 2008), and in line with Guidelines for the welfare and use of animals in cancer research [39].

\section{Drugs and reagents}

Sunitinib (Chemietek) was dissolved in methocel $0.5 \%$ and further diluted in saline solution immediately before use. It was administered daily orally by gavage at the dose of $40 \mathrm{mg} / \mathrm{Kg}$, unless otherwise indicated.

\section{Micro-CT analysis}

Mice were anesthetized with a continuous flow of 3\% isoflurane/oxygen mixture and positioned prone with both legs at right angles. The region spanning the entire torso to the distal tibia of each mouse was scanned with Explore Locus micro-CT scanner (GE Healthcare) without contrast agents. Micro-CT projections of the animals were acquired using $80 \mathrm{kV}, 450 \mu \mathrm{A}$ current, with $100 \mathrm{~ms}$ acquisition time. Four hundred projections were 
acquired over $360^{\circ}$. The resolution of the acquired images was $93 \mu \mathrm{m}$. The reconstructed 3D images were visualized and analyzed using MicroView analysis software (GE Healthcare).

The amount of adipose tissue was determined as described by Luu et al.[40]. Briefly, the gray-scale histogram of the reconstructed images presents a peak that indicates the presence of fat. A low and a high gray-scale threshold corresponding to that peak were chosen. The fat was quantified as the sum of the volumes of all the voxels with a gray-scale value ranging between the low and the high thresholds. The analysis was done on the abdominal region (between the proximal end of L1 and the distal end of S1).

\section{Electron microscopy}

Tibialis Anterior muscles from control, untreated and sunitinib-treated mice were excised at sacrifice, cut in the sagittal plane with a razor blade and fixed with 4\% paraformaldehyde (PFA) and 2\% glutaraldehyde in phosphate buffer $0.12 \mathrm{M}, \mathrm{pH} 7.4$ for 2 hours at $4^{\circ} \mathrm{C}$, followed by incubation at room temperature for $2 \mathrm{~h}$ in OsO4. After dehydration, tissue samples were cleared in propylene oxide, embedded in epoxy medium (Epon 812 Fluka) and polymerized at $60^{\circ} \mathrm{C}$ for $72 \mathrm{~h}$. From each sample, ultrathin (60 $\mathrm{nm}$ thick) sections of areas of interest were obtained, counterstained with uranyl acetate and lead citrate, and examined with an Energy Filter Transmission Electron Microscope (EFTEM, ZEISS LIBRA® ${ }^{\circledR} 120$ ) equipped with a YAG scintillator slow-scan CCD camera.

\section{Muscle fiber cross-sectional area measurements}

Eight- $\mu \mathrm{m}$ depth cross-sectional tissue sections from the TA muscle were fixed in cold acetone, stained with hematoxylin and eosin solutions (Fluka) and digitally imaged with a CKX41 microscope (Olympus). For each muscle, at least 8 randomly selected $20 \mathrm{X}$ magnification images were quantified with $\mathrm{Cell}^{\mathrm{F}}$ software (Olympus).

\section{Western Blot analysis}

Proteins from TA muscles were extracted with a lysis buffer supplemented with proteases inhibitor cocktail (Roche), quantitated by BCA Protein or Coomassie Plus Assay Kit (Pierce), separated by SDS-PAGE and transferred to a PVDF membrane (Millipore). Membranes were probed with mouse anti-MuRF-1 (1:30), rabbit antiatrogin-1 (1:2000, a kind gift of Dr. S. Lecker from Beth Israel Deaconess Medical Center in Boston, USA), rabbit anti-phospho STAT3 (Tyr705, 1:2000, Cell Signaling), mouse anti-STAT3 (1:1000, Cell Signaling) and mouse anti-GAPDH (1:40000, Sigma Aldrich) antibodies. The signal was detected with CDP-Star ${ }$ Substrate (Life Technologies). The MuRF-1 antibody was raised against rat MuRF-1, as previously described [15].

\section{Statistical analysis}

Statistical analyses were done using Prism Software (GraphPad Prism 5.01). Differences in survival were analyzed by the log-rank test. Correlation was assessed by a standard Pearson correlation, after the symmetry of the distribution was assured with Skewness and Kurtosis. Differences among groups of non-time-related measurements were assessed by one-way ANOVA followed by the Bonferroni post-test.

In the figures, significance is indicated as follows: * $\mathrm{P}<0.05, * * \mathrm{P}<0.01, * * * \mathrm{P}<0.001$.

\section{ACKNOWLEDGEMENTS}

This work was supported by the Italian Association for Cancer Research (AIRC n ${ }^{\circ}$. IG14532 and $n^{\circ} .12812$ to RG and AIRC Start-UP n ${ }^{\circ} .11423$ to RP), Fondazione CARIPLO (no. 2009-2626 to CG) and the Union under the Marie Curie International Reintegration Grant (PIRG08GA-2010-277008 to RP). We are grateful to Viviana Rossi for secretarial assistance.

\section{Conflict of interest}

The authors declare no potential conflict of interest.

\section{Abbreviations}

RCC, renal cell carcinoma; TKI, tyrosine kinase inhibitor; MuRF-1, Muscle RING Finger-1 protein; BW, body weight; BWL, body weight loss; TW, tumor weight; MST, median survival time; ILS, increment of life span; TA, tibialis anterior; CSA, cross-sectional area; p-STAT3, phosphorylated STAT3; IL-6, interleukin-6; VEGF, vascular endothelial growth factor; TNF $\alpha$, tumor necrosis factor $\alpha$.

\section{REFERENCES}

1. Fearon K, Strasser F, Anker SD, Bosaeus I, Bruera E, Fainsinger RL, Jatoi A, Loprinzi C, MacDonald N, Mantovani G, Davis M, Muscaritoli M, Ottery F, et al. Definition and classification of cancer cachexia: an international consensus. Lancet Oncol. 2011; 12(5):489495.

2. Fearon KC, Glass DJ and Guttridge DC. Cancer cachexia: mediators, signaling, and metabolic pathways. Cell Metab. 2012; 16(2):153-166.

3. Piccirillo R, Demontis F, Perrimon N and Goldberg AL. 
Mechanisms of muscle growth and atrophy in mammals and Drosophila. Dev Dyn. 2014; 243(2):201-215.

4. Egerman MA and Glass DJ. Signaling pathways controlling skeletal muscle mass. Crit Rev Biochem Mol Biol. 2014; 49(1):59-68.

5. Fearon K, Arends J and Baracos V. Understanding the mechanisms and treatment options in cancer cachexia. Nat Rev Clin Oncol. 2013; 10(2):90-99.

6. Mantovani G, Madeddu C and Maccio A. Drugs in development for treatment of patients with cancer-related anorexia and cachexia syndrome. Drug Des Devel Ther. 2013; 7:645-656.

7. Ding GX, Feng CC, Song NH, Fang ZJ, Xia GW, Jiang HW, Hua LX and Ding Q. Paraneoplastic symptoms: Cachexia, polycythemia, and hypercalcemia are, respectively, related to vascular endothelial growth factor (VEGF) expression in renal clear cell carcinoma. Urol Oncol. 2012.

8. Oya M. Renal cell carcinoma: biological features and rationale for molecular-targeted therapy. Keio J Med. 2009; 58(1):1-11.

9. van der Veldt AA, Haanen JB, van den Eertwegh AJ and Boven E. Targeted therapy for renal cell cancer: current perspectives. Discov Med. 2010; 10(54):394-405.

10. Motzer RJ, Rini BI, Bukowski RM, Curti BD, George DJ, Hudes GR, Redman BG, Margolin KA, Merchan JR, Wilding G, Ginsberg MS, Bacik J, Kim ST, et al. Sunitinib in patients with metastatic renal cell carcinoma. JAMA. 2006; 295(21):2516-2524.

11. Escudier B and Kataja V. Renal cell carcinoma: ESMO Clinical Practice Guidelines for diagnosis, treatment and follow-up. Ann Oncol. 2010; 21 Suppl 5:v137-139.

12. Singer EA, Gupta GN and Srinivasan R. Targeted therapeutic strategies for the management of renal cell carcinoma. Curr Opin Oncol. 2012; 24(3):284-290.

13. Argiles JM, Busquets S, Stemmler B and Lopez-Soriano FJ. Cancer cachexia: understanding the molecular basis. Nat Rev Cancer. 2014; 14(11):754-762.

14. Penna F, Costamagna D, Fanzani A, Bonelli G, Baccino FM and Costelli P. Muscle wasting and impaired myogenesis in tumor bearing mice are prevented by ERK inhibition. PLoS One. 2010; 5(10):e13604.

15. Bodine SC, Latres E, Baumhueter S, Lai VK, Nunez L, Clarke BA, Poueymirou WT, Panaro FJ, Na E, Dharmarajan K, Pan ZQ, Valenzuela DM, DeChiara TM, et al. Identification of ubiquitin ligases required for skeletal muscle atrophy. Science. 2001; 294(5547):1704-1708.

16. Gomes MD, Lecker SH, Jagoe RT, Navon A and Goldberg AL. Atrogin-1, a muscle-specific F-box protein highly expressed during muscle atrophy. Proc Natl Acad Sci U S A. 2001; 98(25):14440-14445.

17. Bonetto A, Aydogdu T, Jin X, Zhang Z, Zhan R, Puzis L, Koniaris LG and Zimmers TA. JAK/STAT3 pathway inhibition blocks skeletal muscle wasting downstream of IL-6 and in experimental cancer cachexia. Am J Physiol
Endocrinol Metab. 2012; 303(3):E410-421.

18. Escudier B, Eisen T, Stadler WM, Szczylik C, Oudard S, Siebels M, Negrier S, Chevreau C, Solska E, Desai AA, Rolland F, Demkow T, Hutson TE, et al. Sorafenib in advanced clear-cell renal-cell carcinoma. N Engl J Med. 2007; 356(2):125-134.

19. Aulino P, Berardi E, Cardillo VM, Rizzuto E, Perniconi B, Ramina C, Padula F, Spugnini EP, Baldi A, Faiola F, Adamo S and Coletti D. Molecular, cellular and physiological characterization of the cancer cachexiainducing C26 colon carcinoma in mouse. BMC Cancer. 2010; 10:363.

20. Hudes G, Carducci M, Tomczak P, Dutcher J, Figlin R, Kapoor A, Staroslawska E, Sosman J, McDermott D, Bodrogi I, Kovacevic Z, Lesovoy V, Schmidt-Wolf IG, et al. Temsirolimus, interferon alfa, or both for advanced renal-cell carcinoma. N Engl J Med. 2007; 356(22):22712281.

21. Wang XH and Mitch WE. Muscle wasting from kidney failure-a model for catabolic conditions. Int J Biochem Cell Biol. 2013; 45(10):2230-2238.

22. Acharyya S, Ladner KJ, Nelsen LL, Damrauer J, Reiser PJ, Swoap S and Guttridge DC. Cancer cachexia is regulated by selective targeting of skeletal muscle gene products. J Clin Invest. 2004; 114(3):370-378.

23. Cohen S, Brault JJ, Gygi SP, Glass DJ, Valenzuela DM, Gartner C, Latres E and Goldberg AL. During muscle atrophy, thick, but not thin, filament components are degraded by MuRF1-dependent ubiquitylation. J Cell Biol. 2009; 185(6):1083-1095.

24. Lagirand-Cantaloube J, Cornille K, Csibi A, BatonnetPichon S, Leibovitch MP and Leibovitch SA. Inhibition of atrogin-1/MAFbx mediated MyoD proteolysis prevents skeletal muscle atrophy in vivo. PLoS One. 2009; 4(3):e4973.

25. Sandri M, Sandri C, Gilbert A, Skurk C, Calabria E, Picard A, Walsh K, Schiaffino S, Lecker SH and Goldberg AL. Foxo transcription factors induce the atrophy-related ubiquitin ligase atrogin-1 and cause skeletal muscle atrophy. Cell. 2004; 117(3):399-412.

26. Stitt TN, Drujan D, Clarke BA, Panaro F, Timofeyva Y, Kline WO, Gonzalez M, Yancopoulos GD and Glass DJ. The IGF-1/PI3K/Akt pathway prevents expression of muscle atrophy-induced ubiquitin ligases by inhibiting FOXO transcription factors. Mol Cell. 2004; 14(3):395-403.

27. Argiles JM, Busquets S, Toledo M and Lopez-Soriano FJ. The role of cytokines in cancer cachexia. Curr Opin Support Palliat Care. 2009; 3(4):263-268.

28. Bonetto A, Aydogdu T, Kunzevitzky N, Guttridge DC, Khuri S, Koniaris LG and Zimmers TA. STAT3 activation in skeletal muscle links muscle wasting and the acute phase response in cancer cachexia. PLoS One. 2011; 6(7):e22538.

29. Zhang L, Pan J, Dong Y, Tweardy DJ, Garibotto G and Mitch WE. Stat3 activation links a C/EBPdelta to myostatin 
pathway to stimulate loss of muscle mass. Cell Metab. 2013; 18(3):368-379.

30. Tierney MT, Aydogdu T, Sala D, Malecova B, Gatto S, Puri PL, Latella L and Sacco A. STAT3 signaling controls satellite cell expansion and skeletal muscle repair. Nat Med. 2014; 20(10):1182-1186.

31. Tsoli M, Schweiger M, Vanniasinghe AS, Painter A, Zechner R, Clarke S and Robertson G. Depletion of white adipose tissue in cancer cachexia syndrome is associated with inflammatory signaling and disrupted circadian regulation. PLoS One. 2014; 9(3):e92966.

32. Zhou X, Wang JL, Lu J, Song Y, Kwak KS, Jiao Q, Rosenfeld R, Chen Q, Boone T, Simonet WS, Lacey DL, Goldberg AL and Han HQ. Reversal of cancer cachexia and muscle wasting by ActRIIB antagonism leads to prolonged survival. Cell. 2010; 142(4):531-543.

33. Baumgarten AJ, Fiebig HH and Burger AM. Molecular analysis of xenograft models of human cancer cachexia-possibilities for therapeutic intervention. Cancer Genomics Proteomics. 2007; 4(3):223-231.

34. Xue Y, Religa P, Cao R, Hansen AJ, Lucchini F, Jones B, Wu Y, Zhu Z, Pytowski B, Liang Y, Zhong W, Vezzoni $\mathrm{P}$, Rozell B, et al. Anti-VEGF agents confer survival advantages to tumor-bearing mice by improving cancerassociated systemic syndrome. Proc Natl Acad Sci U S A. 2008; 105(47):18513-18518.

35. Wong AK, Alfert M, Castrillon DH, Shen Q, Holash J, Yancopoulos GD and Chin L. Excessive tumor-elaborated VEGF and its neutralization define a lethal paraneoplastic syndrome. Proc Natl Acad Sci U S A. 2001; 98(13):74817486.

36. Wang X, Crowe PJ, Goldstein D and Yang JL. STAT3 inhibition, a novel approach to enhancing targeted therapy in human cancers (review). Int J Oncol. 2012; 41(4):11811191.

37. Gilabert M, Calvo E, Airoldi A, Hamidi T, Moutardier V, Turrini $\mathrm{O}$ and Iovanna J. Pancreatic cancer-induced cachexia is Jak2-dependent in mice. J Cell Physiol. 2014; 229(10):1437-1443.

38. Naito S, von Eschenbach AC, Giavazzi R and Fidler IJ. Growth and metastasis of tumor cells isolated from a human renal cell carcinoma implanted into different organs of nude mice. Cancer Res. 1986; 46(8):4109-4115.

39. Workman P, Aboagye EO, Balkwill F, Balmain A, Bruder G, Chaplin DJ, Double JA, Everitt J, Farningham DA, Glennie MJ, Kelland LR, Robinson V, Stratford IJ, et al. Guidelines for the welfare and use of animals in cancer research. Br J Cancer. 2010; 102(11):1555-1577.

40. Luu YK, Lublinsky S, Ozcivici E, Capilla E, Pessin JE, Rubin CT and Judex S. In vivo quantification of subcutaneous and visceral adiposity by micro-computed tomography in a small animal model. Med Eng Phys. 2009; 31(1):34-41. 